\title{
Voice quality after thyroplasty type I using a silicone block
}

\author{
Sebova $\mathrm{I}^{1}$, Ziethe $\mathrm{A}^{2}$, Doellinger $\mathrm{M}^{2}$, Eysholdt $\mathrm{U}^{3}$ \\ Department for Otorhinolaryngology and Head and Neck Surgery, Faculty of Medicine, Comenius University \\ and University Hospital, Bratislava, Slovakia. sebovairina95@mail.com
}

\begin{abstract}
AIM: The aim of the work was to evaluate the voice quality of 10 adult patients after thyroplasty type I using a silicone block. Preoperatively patients suffered from unilateral vocal fold paralysis.

MATERIAL AND METHODS: We evaluated selected preoperative and postoperative patient findings (RBH according to Wendler classification, videolaryngostroboscopy and maximum phonation time MPT). The evaluation was performed by a phoniatrician and clinical speech therapist, using patient medical records and the Lingwaves and Glottis Analysis Tools (GAT) programs.

RESULTS: Preoperatively we identified R1B1H1 - R3B3H3, postoperatively R0B0H0 - R3B3H2. In six cases the paralysed vocal cord was preoperatively in a paramedian position, twice in intermediate position and twice in a lateral position. In the case of 6 patients presbyphonic changes were present. Motility of the healthy vocal cord was within the norm. Postoperatively, the paretic vocal cord was medialised. We assessed the MPT value: preoperatively 3-10 seconds, postoperatively 9-17 seconds. Postoperatively we measured jitter (\%), shimmer (\%) and closing quotient values. In comparison with healthy subjects (Inwald et al. 2011), jitter and shimmer values were higher and closing quotient values after thyroplasty type I close to normal values (Tab.1). CONCLUSIONS: The postoperative voice quality was significantly better in comparison with the preoperative state; though, it never achieved the parameters of a normal voice. We confirmed the significant contribution of TPL I for the patients' quality of life through partial rehabilitation of their voices. Text in PDF www.elis.sk. KEY WORDS: thyroplasty type I, unilateral vocal fold paralysis, voice quality, acoustic analysis, aerodynamic analysis.
\end{abstract}

\section{Introduction}

Thyroplasty type I (hereinafter TPL I) is a surgical method for solving inadequate closure of the vocal cord, which is a cause of significant dysphonia through to aphonia for the patient $(1,4)$. It was performed for the first time by Payr in 1915 (2). It is used primarily for solving unilateral vocal cord paresis; rarely for solving presbyphony, congenital developmental laryngeal defects, or states after laser resection of the vocal cord. Various materials (cartilage, fascia, hyaluronic acid, hydroxylapatite, GORE-TEX, titanium and others) are used for medialisation of the vocal cord. The Division of Phoniatrics and Pediatric Audiology at the Department of Otorhinolaryngology, Head and Neck Surgery, FAU Erlangen-Nuernberg (Federal Republic of Germany) uses the classic TPL I method, using an individually prepared silicone block. The implant is inserted under the paretic vocal cord from an ex-

${ }^{1}$ Department for Otorhinolaryngology and Head and Neck Surgery, Faculty of Medicine, Comenius University and University Hospital Bratislava, Slovakia, ${ }^{2}$ Division of Phoniatrics and Pediatric Audiology at the Department of Otorhinolaryngology, Head and Neck Surgery, FAU Erlangen Nürnberg, University Hospital Erlangen, Federal Republic of Germany, and ${ }^{3}$ Medical Physics Carl von Ossietzky University of Oldenburg, Federal Republic of Germany

Address for correspondence: I. Šebová, MD, PhD, Department for Otorhinolaryngology, Head and Neck Surgery, Faculty of Medicine, Comenius University and University Hospital Bratislava, Antolská 11, SK-851 07 Bratislava, Slovakia. ternal approach; the procedure can be performed under tumescent or general anaesthesia.

\section{Material and methods}

In the work we evaluated the voice quality of 10 patients after TLP I, based on selected parameters (RBH classification according to Wendler, videolaryngostroboscopic finding and maximum phonation time MPT) after the procedure. Patients were operated at the University Hospital in Erlangen (Federal Republic of Germany). We retrieved the data retrospectively from their health documentation. We determined the RBH classification and MPT, while other parameters were measured using the Lingwaves program by a clinical speech therapist. Videolaryngostroboscopic findings and Glottal Area Waveform (GAW) analyses were performed and evaluated by a phoniatrician.

\section{Results}

Ten patients were included in the group, of whom 5 were men and 5 women. In seven cases, this concerned paresis of the left vocal cord, in three cases paresis of the right vocal cord. In six cases, the paralysed vocal cords were preoperatively in a paramedian position, twice in an intermediate position and twice in lateral position. Motivity of the healthy vocal cord was in all cases within the norm, though 6 patients exhibited mild to extensive presbyphonic changes in line with mild to extensive atrophy 
Tab. 1. The objective voice quality analysis (closed quotient, shimmer, jitter) of patiensts after TPL I using GAW program.

\begin{tabular}{|c|c|c|c|c|c|c|}
\hline & \multicolumn{2}{|c|}{$\begin{array}{l}\text { Healthy subject group } \\
\text { Quote: Inwald et al (2011) (6) }\end{array}$} & \multicolumn{2}{|c|}{$\begin{array}{l}\text { Unilateral vocal fold palsy } \\
\text { preoperatively } \\
\text { Quote: Inwald et al (2011) (6) }\end{array}$} & \multicolumn{2}{|c|}{$\begin{array}{l}\text { After thyroplasty type I } \\
\text { Our results }\end{array}$} \\
\hline & Women & Men & Women & Men & Women & Men \\
\hline Closing quotient & $0.37 \pm 0.16$ & $0.53 \pm 0.12$ & $0.22 \pm 0.21$ & $0.21 \pm 0.19$ & $0.40 \pm 0.13$ & $0.45 \pm 0.07$ \\
\hline Shimmer (\%) & $2 \pm 1$ & $3 \pm 1$ & $6 \pm 12$ & $9 \pm 18$ & $4 \pm 3$ & $9 \pm 9$ \\
\hline Jitter (\%) & $4 \pm 2$ & $3 \pm 2$ & $6 \pm 10$ & $8 \pm 16$ & $4.28 \pm 1.57$ & $7.14 \pm 5.22$ \\
\hline
\end{tabular}

of the m.vocalis with excavation. The age of the patients ranged from 32 to 68 years; the average age was 54 years. The average time since the operation was 1.6 years, the shortest being 1 year.

In evaluating the voice by hearing, we found, based on the RBH classification, preoperatively R1B1H1 - R3B3H3, postoperatively $\mathrm{R} 0 \mathrm{~B} 0 \mathrm{H} 0$ - $\mathrm{R} 3 \mathrm{~B} 3 \mathrm{H} 2$. All patients subjectively reported significant voice improvement (Fig. 1).

With the aid of a videolaryngoscopic examination we found: - preoperatively: shift of vocal cord edge $-1 \mathrm{x}$ moderately reduced and $7 \mathrm{x}$ absent, symmetry and regularity - moderate to severe asymmetry and irregularity, vocal fold closure-moderately to completely disturbed, shape of incomplete vocal fold closure $-7 \mathrm{x}$ longitudinal and $1 \mathrm{x}$ spindle, supraglottic compensation - in 8 cases mild to severe. - postoperatively: shift of vocal cord edge $-2 x$ normal, $3 x$ mild to moderately reduced and $3 \mathrm{x}$ absent, symmetry $-7 \mathrm{x}$ mild / moderate

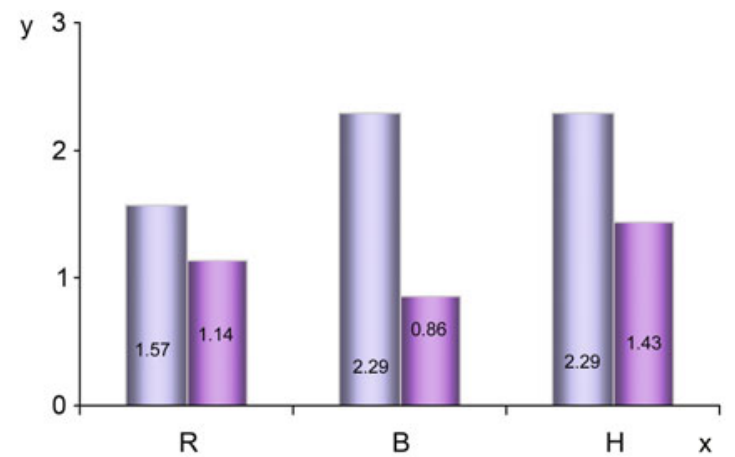

Fig. 1. Postoperative voice quality according to $\mathrm{RBH}$ classification by Wendler.

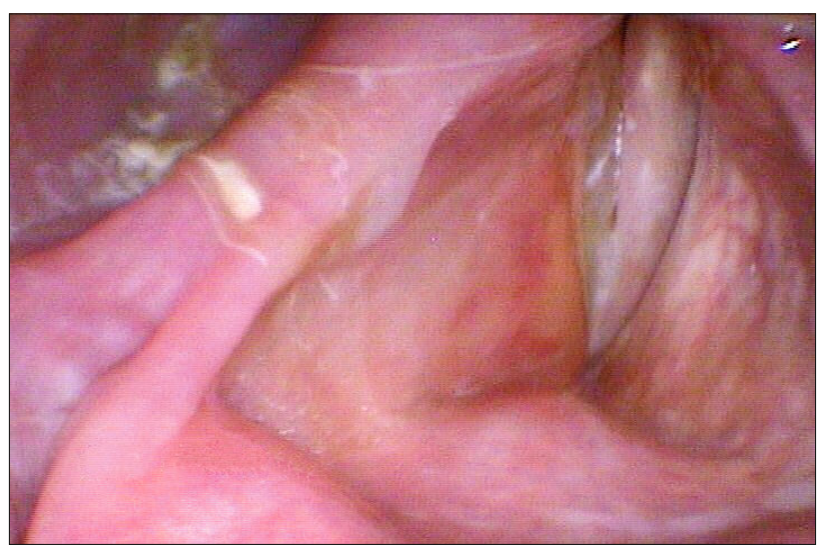

Fig. 2. State after unilateral paresis of the left vocal cord, state after TPL I, the medialised vocal cord is in the middle line, the vocal cord closure is complete. asymmetry and $1 \mathrm{x}$ severe asymmetry, regularity $-5 \mathrm{x}$ normal and $3 \mathrm{x}$ mild to severe irregularity, vocal fold closure $-5 \mathrm{x}$ complete (Fig. 2), 3x dorsal insufficiency, supraglottic compensation - mild to severe in 8 cases.

In conclusion, in the aerodynamics assessment we investigated the value of the maximum phonation time, the values of which were preoperatively 3-10 seconds, postoperatively 9-17 seconds.

After the operation, we examined each patient in the framework of an objective voice quality analysis using High Speed Videoendoscopy (HSVE) (Fig. 3) and the Lingwaves program, extracting Glottal Area Waveform (GAW) and analysing shimmer (\%), jitter (\%) and closing quotient values. In comparison with healthy subjects (Inwald et al. 2011) (6), jitter and shimmer values were higher and closing quotient values after thyroplasty I were close to normal values (Tab.1).

We examined the movement of the vocal cords using a special camera inserted transorally above the larynx entrance, and we recorded a two-second video during phonation. This recording can be viewed with the naked eye in slow motion. An advantage of this is that, unlike stroboscopy, we do not lose any moment from the vocal cord movement - we also see irregular oscillations. Using the Glottis Aalysis Tools (GAT) program, we can objectively calculate the vocal cord movement parameters (5) from the recording.

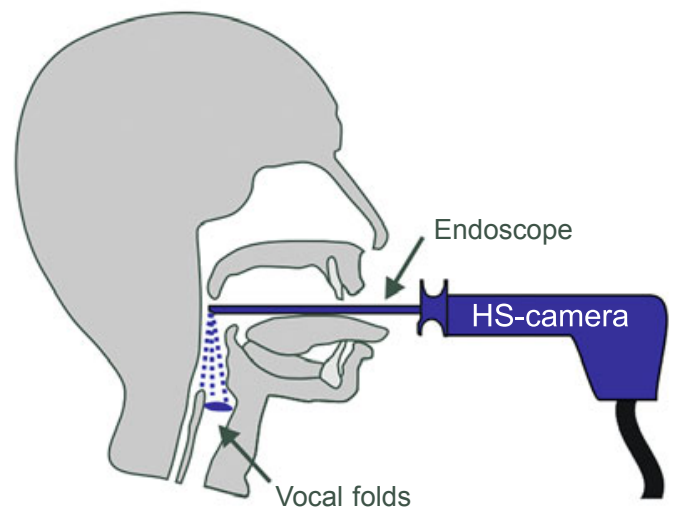

Fig. 3. Technique of the High Speed Videoendoscopy (HSVE). We examined the vocal cords using a special camera inserted transorally above the larynx entrance, and we recorded a two-second video during phonation. This recording can be viewed with the naked eye in slow motion. An advantage of this is that, unlike stroboscopy, we do not lose any moment from the vocal cord movement - we also see irregular oscillations. Using the Glottis Area Waveform (GAW) program, we can objectively calculate the vocal cord movement parameters from the recording. 


\section{Discussion}

The ELS Voice Diagnostic Protocol defines subjective and objective methods for determining voice quality (3). It serves as a useful tool that enables phonosurgeons to evaluate the results of phonosurgical procedures. We used some recommended methods for this purpose in our work.

Similarly, Ngaetopprutaram et al (2018) evaluated a set of 19 patients in whom unilateral vocal cord paresis was solved by medialisation TPL. Voice recordings were performed before surgery and at 2, 4 and 6 months after surgery. The acoustic analysis (fundamental frequency, highest fundamental frequency, lower fundamental frequency, noise-to-harmonic ratio, and percentage of jitter and shimmer) was calculated using the Multidimensional Voice Program. Maximum phonation time, perceptual evaluation, and a self-assessment visual analogue scale were also conducted. Data for the preoperative and three postoperative periods were compared.

Jitter, shimmer, noise-to-harmonic ratio, and maximum phonation time showed significant improvements after medialization thyroplasty, and maximum improvement was found at the second postoperative month, with no significant change during the three postoperative periods. Subjective voice quality and loudness showed maximum improvement at the fourth and sixth months, respectively. Patients' self-assessment by visual analogue scale showed maximum improvement at the fourth postoperative month, with up to $80 \%$ of their normal voice.

All objective measurements revealed maximum voice improvement at the second month after medialization thyroplasty, with marginal improvement thereafter. The subjective measurements demonstrated an improvement up to $80 \%$ of normal voice after surgery (8).

In clinical practice, there are several ways how to medialise the vocal cord(s). The decision between injection augmentation methods and medialisation of the vocal cord(s) from an external approach is based on the patient's individual circumstances and according to local findings.

Nassimizadeh et al (2019) evaluated 186 patients (a median age of 66 years (interquartile range [IQR]: 51-75), of whom $61 \%$ were male) in whom injection augmentation of the vocal cord(s) was performed for the purpose of their medialisation and improvement of vocal cord closure.

VHI-10 score improved significantly, from a mean of 26.7 to 12.5 ( $\mathrm{p}$.001). A significant improvement in MPT was also observed, from a median of 3.0 to $6.3(n=66, \mathrm{p}<.001)$. Improvements in all components of the GRBAS score were also observed (all $\mathrm{p}<.001$ ), with between $43 \%$ and $88 \%$ of cases reporting reductions after the procedure. Patients receiving a repeated procedure saw a significantly smaller improvement in VHI-10 than those where it was the primary treatment (mean reduction: 9.8 vs 15.5, $\mathrm{p}=.018$ ). Analysis of MPT found a significant correlation between the quantity of injection material used and the degree of improvement observed (rho $=0.355, \mathrm{p}=.004)(7)$.

Our work showed that Isshiki TPL type I using a silicone block is an effective surgical method for solving unilateral vocal cord paresis. Using the selected methods, we found a reduction in perturbation effects. Despite good postoperative vocal cord closure, mild dysphonia persists among patients, based on conditional entrainment. Despite the fact that the procedure only partially solves presbyphonic changes on the side of the affected vocal cord in the elderly, patients were satisfied with their voice and did not require further voice corrections.

\section{Conclusions}

Postoperative voice quality after TPL type I is significantly better in comparison with the preoperative state, yet never achieves, either subjectively or objectively, normal voice parameters. Compared to injection medialisation techniques, its results are long-term. Subjective evaluation of the voice by the patient is generally better than objective evaluation of the patient's voice by a phonosurgeon or clinical speech therapist. It is advisable to carry out an assessment of the voice according to the basic protocol recommended by the European Laryngological Society (3), as this leads to standardisation of the evaluation and allows comparison of results between individual workplaces.

\section{References}

1. Bihary A, Mészáros K, Reményi A, Lichtenberger G. Voice quality improvement after management of unilateral vocal cord paralysis with different techniques. Eur Arch Otorhinolaryngol 2006; 263: 1115-1120.

2. Crolley VE, Gibbins N. One hundred years of external approach medialisation thyroplasty. J Laryngol Otol 2017; 131 (3): 202-208.

3. Dejonckere PH, Bradley P, Clemente P, Cornut G, Crevier-Buchman L, Friedrich G, Van de Heyning P, Remacle M, Woisard V. A basic protocol for functional assessment of vocie pathology, especially for investigating the efficacy of (phonosurgical) treatments and evaluating new assement techniques. Guideline elaborated by the Committee on Phoniatrics of the European Laryngological Society (ELS). Eur Arch Otorinolaryngol 2001; 258: 77-82.

4. Friedrich G, Marie JP, Arens Ch. Defining phonosurgery: a proposal for classification and nomenclature by the Phonosurgery Committee of the European Laryngological Society (ELS) Eut Arch Otorhinolaryngol 2007; 264 (1): 191-200.

5. Havla J, Doellinger M, Eysholdt U, Lohscheller J. Repropduzierbarkeit und Reliabilität des Phonovibrogramms (Quantifizierung gesunder Stimmlippenschwingungen). HNO 2009; 57: 160-168.

6. Inwald EC, Doellinger M, Schuster M, Eysholdt U, Bohr Ch. Multiparametric analysis of vocal cord vibrations in healthy and disordered voices in high-speed imaging. J Voice 2011; 25: 576-590.

7. Nassimizadeh A, Shummar SE, Emery K, Costello D. Vocal fold medialization - A 5 year series of single surgeon consecutive medialization with review of literature. J Eval Clin Pract 2019; 1: 1-9.

8. Ngaotepprutaram P, Nakarat T, Aumsompong K, Buakanok N, Chotigavanich C, Chongkolwataba C. Voice improvement after medialization thyroplasty in unilateral vocal fold paralysis. J Med Assoc Thai 2018; 101 (7): 933-938.

Received July 1, 2019. Accepted July 6, 2019. 